\title{
PAPER Strategy for Improving Target Selection Accuracy in Indirect Touch Input
}

\author{
Yizhong XIN ${ }^{\dagger a)}$, Member, Ruonan $\mathrm{LIU}^{\dagger}$, and Yan $\mathrm{LI}^{\dagger \dagger}$, Nonmembers
}

\begin{abstract}
SUMMARY Aiming at the problem of low accuracy of target selection in indirect touch input, an indirect multi-touch input device was designed and built. We explored here four indirect touch input techniques which were TarConstant, TarEnlarge, TarAttract, TarEnlargeAttract, and investigated their performance when subjects completing the target selection tasks through comparative experiments. Results showed that TarEnlargeAttract enabled the shortest movement time along with the lowest error rate, $2349.9 \mathrm{~ms}$ and $10.9 \%$ respectively. In terms of learning effect, both TarAttract and TarEnlargeAttract had learning effect on movement time, which indicated that the speed of these two techniques can be improved with training. Finally, the strategy of improving the accuracy of indirect touch input was given, which has reference significance for the interface design of indirect touch input.
\end{abstract}

key words: indirect touch input, target selection, accuracy, learning effect

\section{Introduction}

In recent years, touch input has been popular in many situations because of its natural and intuitive way of operation. However, this way of direct contact with the screen has some limitations. Firstly, the user's finger or arm may occlude part of the screen. Secondly, in the process of touch input, the resolution of the finger as an indicator device is too low to point to the target less than the finger width [1], which reduce the accuracy of target selection. Therefore, some researchers had proposed indirect touch input to overcome these limitations by creating more complex indirect mapping between control and display space [2]. However, there are also some problems with indirect touch input. When the user selected the target, the accuracy of the touch may be lowered due to the inconsistency of the display position and the operation position.

Aiming at the problem of the low accuracy of indirect touch input, four indirect input techniques were designed. The influence of different input techniques on the accuracy of indirect touch input was compared and investigated by empirical means, and the interactive design suggestions for improving the accuracy of indirect multi-touch input were given.

\section{Manuscript received August 5, 2019.}

Manuscript revised March 2, 2020.

Manuscript publicized April 10, 2020.

†The authors are with Shenyang University of Technology, Shenyang, 110142, China.

${ }^{\dagger \dagger}$ The author is with Shenyang Sport University, Shenyang, 110102, China.

a) E-mail: xyz@sut.edu.cn

DOI: 10.1587/transinf.2019EDP7218

\section{Related Work}

\subsection{Multi-Touch Input}

In 1982, Nimish Mehta of the University of Toronto designed the first multi-touch interface "Flexible Machine Interface" [3] that sensed the pressure of the index finger. In 1991, a major breakthrough was made in multi-touch technology. Wellnerd [4] proposed a touch-screen technique called "Digital Desktop" that allowed users to touch multiple fingers and pulled images within the touch screen at the same time. In 2001, Dietz et al. [5] designed a multitouch sensing input device called "Diamond Touch", which used vertical and horizontal sensors to detect finger positions and allowed simultaneous interaction between multiple users. In 2002, Rekimoto et al. [6] proposed "SmartSkin" multi-touch system that can recognize the contact position and shape of multiple fingers, and can calculate the distance between the hand and the contact surface by capacitance induction and grid antenna. In 2005, Han [7] proposed a multi-touch device based on FTIR (Frustrated Total Internal Reflection) technique. In 2006, Benko et al. [8] proposed five techniques to help users make small target selections on multi-touch devices. In 2008, Jo [9] proposed a sound drawing tool "DrawSound" utilizing multi-touch input technology. In 2009, Hancock et al. [10] proposed an interactive paradigm based on 6DOF on multi-touch devices to achieve interaction freedom. In 2010, Zeleznik et al. [11] proposed a multi-touch system called "Hands-On Math" to help users recognize mathematical symbols or manipulate mathematical expressions. In 2011, Sangsuriyachot et al. [12] proposed a novel interaction technique that combined hands and feet to enhance multi-touch interaction. In 2012, Oakley et al. [13] compared graphical password input based on multi-touch to traditional password input and found that the performance of multi-touch input was related to the user's perception. In 2017, Liu et al. [14] proposed CoReach technology to achieve multi-touch operation between multiple users. In 2018, Nittala et al. [15] proposed a skin-based multi-touch input technology.

\subsection{Direct and Indirect Touch Input}

Forlines et al. [16] investigated the effects of direct singlefinger touch and mouse click on target selection speed and accuracy, and found that the target selection error rate and 
target selection time for direct single-finger touch were higher than those for mouse clicks. Vogel et al. [17] proposed "Shift technique" to eliminate the occlusion in direct touch by creating a callout that displayed the occluded text content and placed it in a non-occluded position. Wang et al. [18] proposed an algorithm to investigate the role of finger pointing in touch interaction by real-time detecting the direction vector of finger dropping, which was instructive to direct touch interface direction perception interaction. Schmidt et al. [19] investigated the direct multi-touch mode that the inputs and outputs were in the same place and the indirect multi-touch mode that the inputs and outputs were not in the same place, and found that direct multi-touch was faster than indirect multi-touch. Pfeuffer et al. [20] proposed a bimodal input technique which combined pen and touch technology to facilitate eye tracking operation in direct and indirect touch input. Voelker et al. [21] compared absolute and relative gaze augmented selection techniques to traditional direct touch methods and found that the relative gaze augmented selection technology was superior to the other technologies. Palleis et al. [22] investigated the effects of indirect touch input on spatial memory and navigation performance in a $2 \mathrm{D}$ panning task. The results showed that indirect touch input did not affect the performance of spatial memory and navigation. Pfeuffer et al. [23] proposed the "CursorShift" technique that allows users to switch between direct-touch input and cursor input. Palleis et al. [24] proposed a two-handed interaction technique to display direct and indirect touch inputs in an interactive workspace. Palleis et al. [25] investigated the indirect touch input through onefinger dragging, two-finger pinch-to-zoom, and rotation gestures, and found that the operation with both hands was the fastest. Findlater et al. [26] investigated the performance of mouse input and indirect touch input, and found that indirect touch input was faster than mouse input for the users with upper body motor impairments.

\section{Indirect Touch Input Technique Design}

\subsection{The TarConstant Technique}

The TarConstant technique is a baseline reference technique for the performance comparison to the other three techniques. For the TarConstant technique, the subjects are asked to use their fingers to touch the touch panel to select the red targets shown on the screen sequentially. The targets' shapes and locations remain unchanged during the selection process. A black circle is shown as a touch cursor to indicate the touch position when the subject' finger came into contact with the touch panel. The subjects can keep their fingers in touch with the panel and slide their fingers to move the cursor into the targets if their first touches do not bring the cursor into the target (Fig. 1).

\subsection{The TarEnlarge Technique}

According to the Fitts' law [27], the movement time is in-

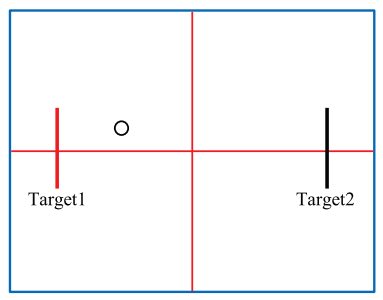

(a) Target1 to be selected

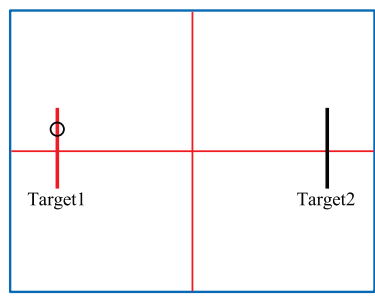

(b) Selected Target1
Fig. 1 The TarConstant technique

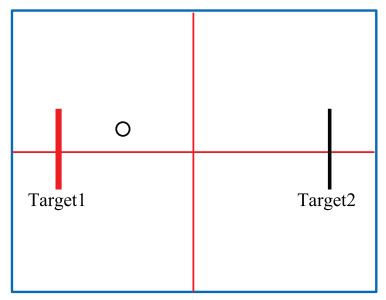

(a) Enlarged Target1

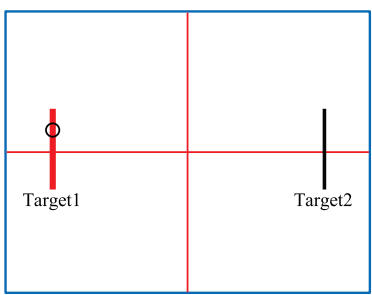

(b) Selected Target1
Fig. 2 The TarEnlarge technique

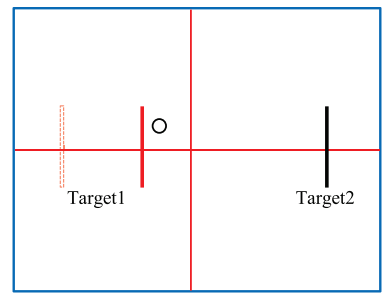

(a) Attracted Target1

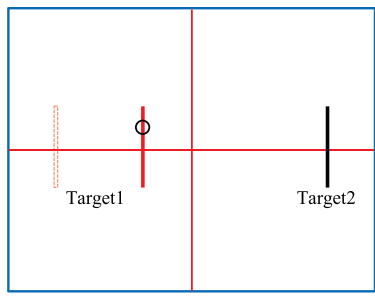

(b) Selected Target1
Fig. 3 The TarAttract technique

versely proportional to the target width. The wider target, the less time is used to select the target. The principle of the technique is to shorten the selection time by increasing the target width. For the TarEnlarge technique, after the subjects' fingers come into contact with the touch panel, the targets are enlarged. This technique provides the subjects with wider target area, which can also alleviate the finger occlusion problem (Fig. 2).

\subsection{The TarAttract Technique}

According to the Fitts' law, the movement time is proportional to the target distance. The shorter target distance, the less time is used to select the target. The principle of the technique is to shorten the selection time by decreasing the target distance. For the TarAttract technique, after the subjects' fingers come into contact with the touch panel, the targets are attracted close to the touch position and the subjects can slide their fingers with a short distance to bring the cursor into the targets. This technique can also reduce the subjects' fatigue (Fig. 3). 


\subsection{The TarEnlargeAttract Technique}

The principle of the technique is to shorten the selection time not only by increasing the target width but also by decreasing the target distance. For the TarEnlargeAttract technique, after the subjects' fingers come into contact with the touch panel, the targets are enlarged and attracted close to the cursor's position. With the technique, the subjects can select the targets more rapidly and easily (Fig. 4).

\section{Task Design}

3 females and 6 males were invited to participate in the experiment. The equipment used in the experiment was an indirect multi-touch device built with optical and computer vision technology (Fig. 5). The subjects were instructed to select the red target on the screen as quickly as accurately as possible through the four indirect touch input techniques on the indirect touch panel. When the subjects' fingers came into contact with the touch panel, a cursor was shown to indicate the fingers' position. If the subjects made a mistake in the process, a failure "ding" sound cue was given to the subject. A within-subject full factorial design with repeated measures was used. The independent variables were target width $(2,4,8,12$ pixels), target distance $(128,256$, 512 pixels) and indirect touch input technique (TarConstant, TarEnlarge, TarAttract, TarEnlargeAttract). In total, the experiment consisted of:

9 subjects $\times 4$ target widths $\times 3$ target distances $\times 4$ indirect touch input techniques $\times 5$ blocks $\times 2$ repetitions $=$ 4320 trials

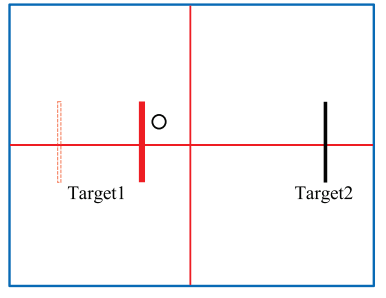

(a) Attracted and Enlarged Target1

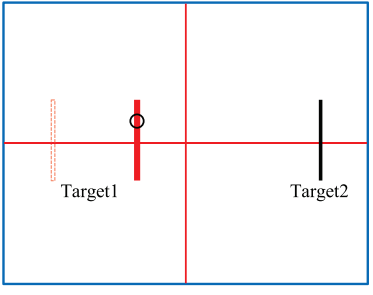

(b) Selected Target1
Fig. 4 The TarEnlargeAttract technique

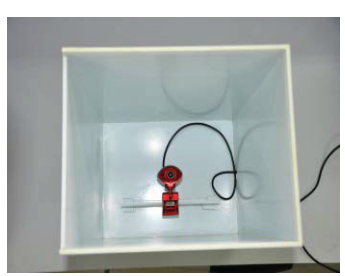

(a) Camera position

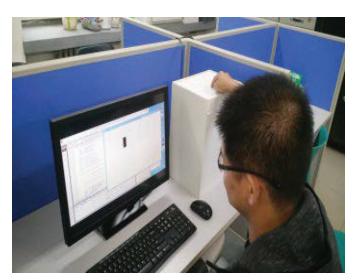

(b) Actual operation process
Fig. 5 Indirect multi-touch device

\section{Results}

\subsection{Movement Time}

Repeated measures analysis of variance showed a significant main effect on movement time for target width $\left(F_{3,24}=\right.$ $39.51, p<.001)$, target distance $\left(F_{2,16}=25.48, p<.001\right)$ and indirect touch input technique $\left(F_{3,24}=39.84, p<.001\right)$. Furthermore, there was also a significant interactive effect on movement time for indirect touch input technique $\times$ target distance $\left(F_{6,48}=7.76, p<.001\right)$. However, there was no significant interactive effect on movement time for target distance $\times$ target width $\left(F_{6,48}=2.06, p=0.08\right)$ and indirect touch input technique $\times$ target width $\left(F_{9,72}=1.61\right.$, $p=0.13$ ). Figure 6 and Fig. 7 illustrate the results.

Figure 6 showed that as the target width increased, the movement time of the four input techniques decreased. Figure 7 showed that as the target distance increased, the movement time for completing the target selection using TarConstant increased significantly. This result was fitted in accordance with the Fitts' law. However, it was worth noting that when TarAttract technique was used to complete the target selection task, the movement time decreased during the increase of the target distance. In addition, when the TarEnlargeAttract technique was used to complete the target selection task, the movement time did not increase significantly during the increase of the target distance.

Among the four indirect touch input techniques, the movement time of TarConstant technique, TarEnlarge tech-

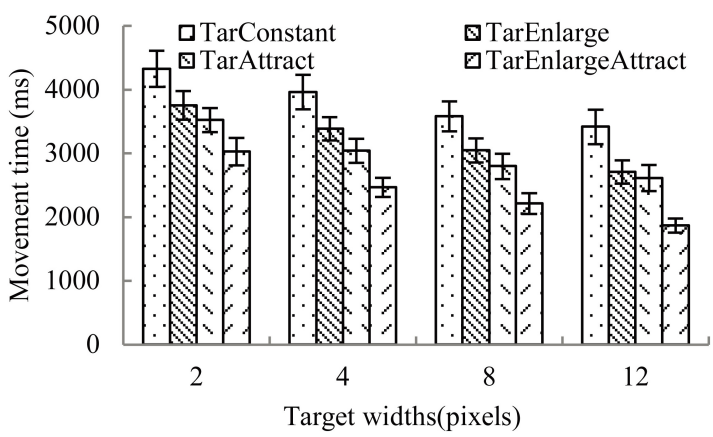

Fig. 6 Movement time per technique $\times$ target width

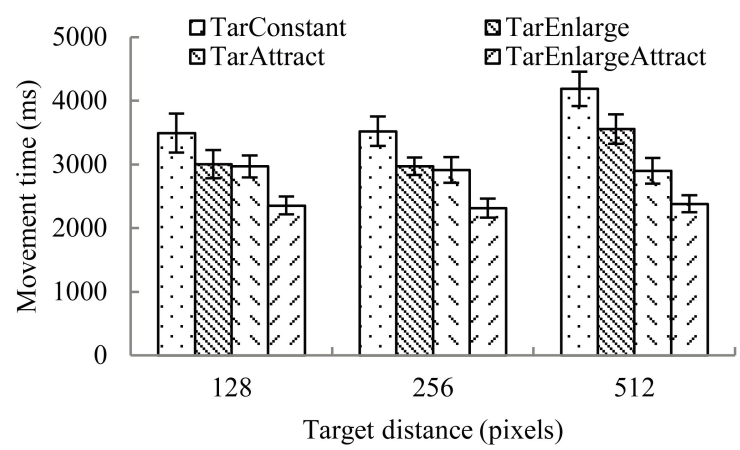

Fig. 7 Movement time per technique $\times$ target distance 


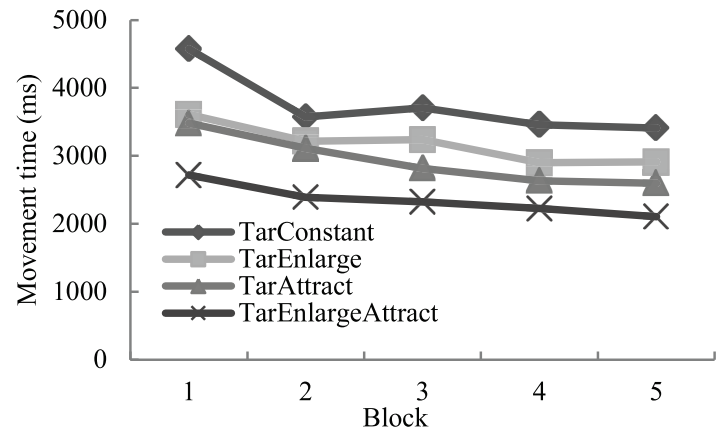

Fig. 8 Movement time per block $\times$ technique

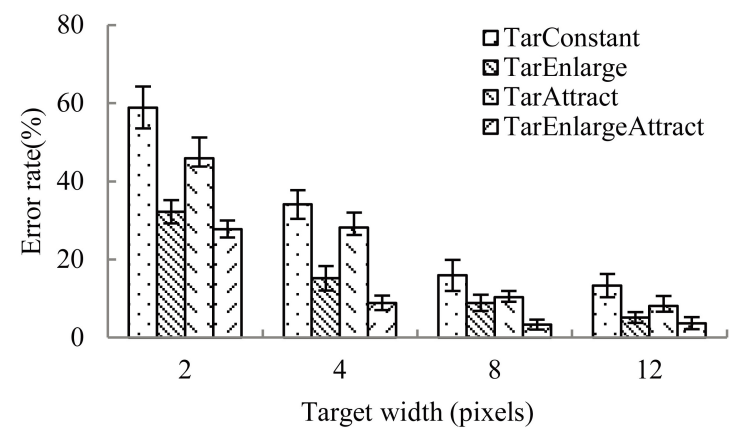

Fig. 9 Error rate per technique $\times$ target width

nique, TarAttract technique and TarEnlargeAttract technique were $3730.3 \mathrm{~ms}, 3178.2 \mathrm{~ms}, 2926.7 \mathrm{~ms}$ and $2349.9 \mathrm{~ms}$ respectively. It can be seen that the TarConstant technique took longer time to complete the target selection task than other techniques, while the TarEnlargeAttract technique took the shortest time.

Repeated measures analysis of variance showed that block number had a significant effect on movement time $\left(F_{4,32}=10.11, p<.001\right)$. With the increase of block number, the movement time of the TarAttract and the TarEnlargeAttract technique was significantly reduced. Figure 8 illustrates the results.

\subsection{Error Rate}

Repeated measures analysis of variance showed a significant effect on error rate for target width $\left(F_{3,24}=83.06\right.$, $p<.001)$ and indirect touch input technique $\left(F_{3,24}=22.41\right.$, $p<.001)$. Furthermore, there was also a significant interactive effect on error rate for indirect touch input technique $\times$ target width $\left(F_{9,72}=4.29, p<.001\right)$. Figure 9 illustrates the results.

Target distance had no significant effect on error rate $\left(F_{2,16}=0.74, p=0.49\right)$. Furthermore, there was no significant interactive effect on error rate for target distance $x$ target width $\left(F_{6,48}=1.13, p=0.36\right)$ and indirect touch input technique $\times$ target distance $\left(F_{6,48}=0.43, p=0.86\right)$. Figure 10 illustrates the results.

Figure 9 showed that as the target width increased, the target selection error rate of the four input techniques was

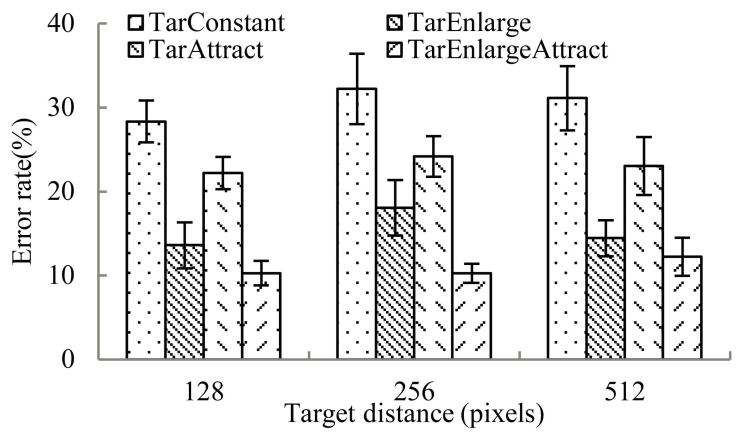

Fig. 10 Error rate per technique $\times$ target distance

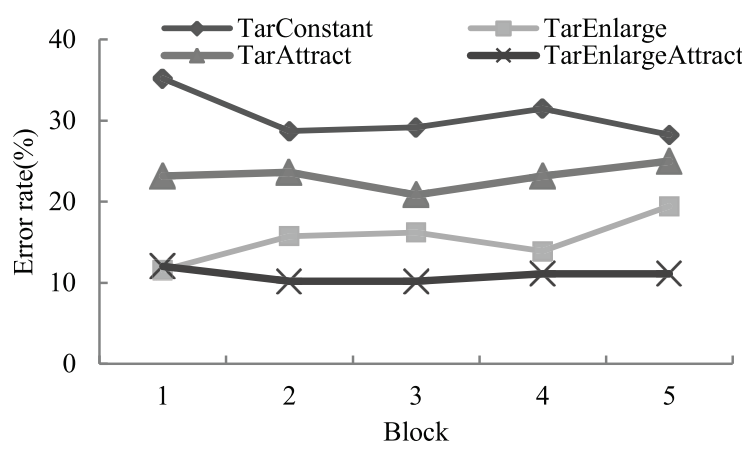

Fig. 11 Error rate per block $\times$ technique

significantly reduced. And Fig. 10 showed that the target selection error rate was the lowest for the four input techniques when the target distance was 128 pixels.

The error rates of TarConstant technique, TarEnlarge technique, TarAttract technique and TarEnlargeAttract technique were $30.6 \%, 15.4 \%, 23.1 \%$ and $10.9 \%$ respectively. It can be seen that the error rate of the TarConstant was higher than other input techniques, and the target selection error rate of the TarEnlargeAttract was the lowest.

Block number had no significant effect on error rate $\left(F_{4,32}=0.18, p=0.95\right)$. Figure 11 illustrates the results.

\subsection{Subjective Evaluation}

After the experiment, the subjects were asked to evaluate the four techniques. Techniques were ranked according to hand comfort, eye comfort, easiness, relaxation, and preference on 7-point Likert scales (Table 1). 1 meant the most negative. 7 meant the most positive. The comprehensive index was examined according to the average of the five evaluation values.

In general, the subjects regarded TarEnlargeAttract as the best technique and TarConstant as the worst technique. The questionnaire survey showed that TarConstant was prone to fatigue in target selection tasks. When the target was extremely small, it was difficult for subjects to concentrate attention for the reason that the subjects had to switch attention between eyes and hands frequently with TarConstant technique. The subjects reported that when the TarAttract technique was used, the finger did not need to 
Table 1 Subjective evaluation

\begin{tabular}{ccccccc}
\hline & Hand Comfort & Eye Comfort & Easiness & Relaxation & Preference & Comprehensive Index \\
\hline TarConstant & 1.11 & 1.11 & 1.40 & 1.67 & 1.78 & 1.41 \\
TarEnlarge & 2.44 & 3.78 & 3.11 & 4.22 & 3.67 & 3.44 \\
TarAttract & 3.00 & 3.22 & 2.67 & 4.00 & 3.00 & 3.18 \\
TarEnlargeAttract & 4.56 & 4.56 & 4.44 & 4.89 & 4.56 & 4.60 \\
\hline
\end{tabular}

move to the target position. They needed only to touch any position on the touch panel, and then the target was attracted close to his finger, which was very convenient.

\section{Discussion}

Among the four indirect touch input techniques, TarEnlargeAttract achieved the best comprehensive performance: the shortest movement time, the lowest error rate and the best subjective evaluation. The TarConstant achieved the worst comprehensive performance: the longest movement time, the highest error rate and the worst subjective evaluation.

The movement time decreased during the increase of the target distance with TarAttract technique. The subjects rarely moved their fingers with TarEnlargeAttract technique so that the movement time of the TarEnlargeAttract technique hardly changed when the target distance increased. The TarEnlargeAttract technique reduced the frequency of attention switches between finger and eye in the process of target selection, and improved the selection speed and reduced fatigue. In terms of learning effect, the movement time of TarAttract and TarEnlargeAttract was significantly decreased, which meant that the speed of these two techniques can be improved rapidly through training. In addition, some subjects argued that the TarEnlarge technique improved the accuracy of target selection and reduced their cognitive load by enlarging the size of the target.

\subsection{Small Target Selection}

Small target selection on touch input devices has always been a vital issue. If the target is smaller than the finger, it is prone to miselection due to difficulty. Although indirect touch input eliminates finger occlusion, the user's attention still switches back and forth between the target on the screen and the touch position on the touch panel. Moreover, when the target is extremely small, the switching frequency is forced to speed up, which deteriorates the selection performance. The proposed techniques in this paper effectively improved the situation particularly for tiny targets (e.g. 2 pixels and 4 pixels targets in the experiment). According to Fig. 9, compared with the TarConstant technique, the TarEnlarge, the TarAttract and the TarEnlargeAttract techniques all improved the accuracy performance. The TarEnlargeAttract technique achieved the best. The error rates of the TarEnlargeAttract technique were 53\%, 74\%, 79\% and

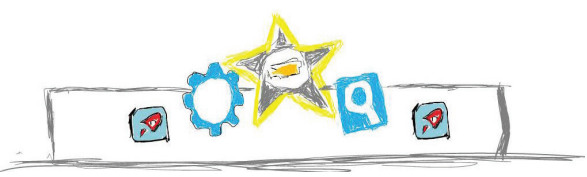

Fig. 12 Enlarged targets

$72 \%$ less than those of the TarConstant technique for target size 2 pixels, 4 pixels, 8 pixels and 12 pixels respectively.

\subsection{Strategy for Improving Target Selection Accuracy in Indirect Touch Input}

\subsubsection{Target Enlargement Strategy}

The various menu items in the WIMP interfaces are often very small and densely arranged, especially when the user operates with indirect touch device such as a notebook's touchpad, which results in more difficulties. The experimental results showed that the error rate of the TarEnlarge and TarEnlargeAttract was significantly lower than the other two techniques, which indicated that the accuracy of indirect touch input could be effectively improved by using target enlargement technique. Although TarEnlarge technique solves the difficult problem of small target selection, this technique may occlude the targets. A possible solution is that when TarEnlarge technique is used, the adjacent targets are enlarged and dispersed away to avoid occlusion (Fig. 12).

\subsubsection{Target Attraction Strategy}

From the experimental results, it can be seen that the performance of TarAttract was better than that of the TarConstant in terms of movement time and error rate, which indicated that attracting the target close to the fingers can improve the accuracy and speed of target selection on indirect touch devices. Therefore, in the interface design of indirect touch input, target attraction method can be used to select remote targets. For example, the menus in the WIMP interfaces are often presented horizontally or vertically. According to the Fitts' law, the longer target distance, the more time is needed to select the target. As a results, there is a lack of fairness for menu items of horizontal/vertical menus. Inspired by the target attraction technology, the menu items can be attracted close to the cursor position (Fig. 13). At all time, a red dot cursor indicates the touch position. When the cursor moves to a certain direction, the menu items in that direction will 


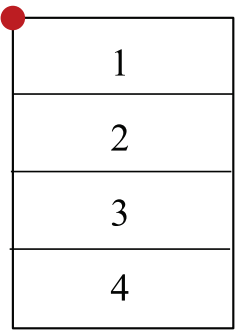

(a) Linear menu layout

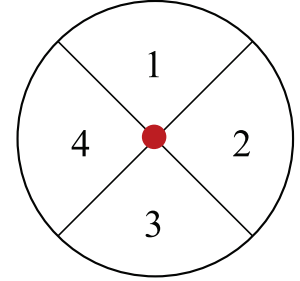

(b) Circle menu layout
Fig. 13 Menu layout

be attracted to the vicinity of the finger, and then the user can select the item with little finger movement.

\section{Conclusion}

Aiming at the low accuracy of target selection in indirect touch devices, an indirect multi-touch input device was designed and built. Four techniques to improve the accuracy of indirect touch input were compared and investigated. The experimental results showed that the TarEnlargeAttract had the best input performance in terms of movement time, error rate and subjective evaluation. TarEnlargeAttract and TarAttract had learning effect on movement time, which indicated that the speed of these two techniques can be improved with training. According to the experimental results, the strategies and design suggestions for improving the accuracy of indirect touch input were given, which has guiding significance for the interface design of indirect multi-touch input.

\section{References}

[1] P.-A. Albinsson and S. Zhai, "High precision touch screen interaction," Proc. SIGCHI 2003, pp.105-112, 2003.

[2] T. Moscovich and J.F. Hughes, "Indirect mappings of multi-touch input using one and two hands," Proc. SIGCHI 2008, pp.1275-1284, 2008.

[3] N. Mehta, "A flexible machine interface," master's thesis, Department of Electrical Engineering, University of Toronto, Toronto, Canada, 1982.

[4] P. Wellner, "Interacting with paper on the digitalDesk," Communications of the ACM, vol.36, no.7, pp.87-96, 1993.

[5] P. Dietz and D. Leigh, "DiamondTouch: a multi-user touch technology," Proc. UIST 2001, pp.219-226, 2001.

[6] J. Rekimoto, "SmartSkin: an infrastructure for freehand manipulation on interactive surfaces," Proc. SIGCHI 2002, pp.113-120, 2002.

[7] J.Y. Han, "Low-cost multi-touch sensing through frustrated total internal reflection," Proc. UIST 2005, pp.115-118, 2005.

[8] H. Benko, A.D. Wilson, and P. Baudisch, "Precise selection techniques for multi-touch screens," Proc. SIGCHI 2006, pp.1263-1272, 2006.

[9] K. Jo, "DrawSound: a drawing instrument for sound performance," Proc. TEI 2008, pp.59-62, 2008.

[10] M. Hancock, T.T. Cate, and S. Carpendale, "Sticky tools: full 6 DOF force-based interaction for multi-touch tables," Proc. ITS 2009, pp.133-140, 2009.

[11] R. Zeleznik, A. Bragdon, F. Adeputra, and H.-S. Ko, "Hands-on math: a page-based multi-touch and pen desktop for technical work and problem solving," Proc. UIST 2010, pp.17-26, 2010.
[12] N. Sangsuriyachot, H. Mi, and M. Sugimoto, "Novel interaction techniques by combining hand and foot gestures on tabletop environments," Proc. ITS 2011, pp.268-269, 2011.

[13] I. Oakley and A. Bianchi, "Multi-touch passwords for mobile device access," Proc. UbiComp 2012, pp.611-612, 2012.

[14] C. Liu, O. Chapuis, M. Beaudouin-Lafon, and E. Lecolinet, "CoReach: cooperative gestures for data manipulation on wall-sized displays," Proc. CHI 2017, pp.6730-6741, 2017.

[15] A.S. Nittala, A. Withana, N. Pourjafarian, and J. Steimle, "Multitouch skin: a thin and flexible multi-touch sensor for on-skin input," Proc. CHI 2018, pp.1-12, 2018.

[16] C. Forlines, D. Wigdor, C. Shen, and R. Balakrishnan, "Directtouch vs. mouse input for tabletop displays," Proc. SIGCHI 2007, pp.647-656, 2007.

[17] D. Vogel and P. Baudisch, "Shift: a technique for operating pen-based interfaces using touch," Proc. SIGCHI 2007, pp.657-666, 2007.

[18] F. Wang, X. Cao, X. Ren, and P. Irani, "Detecting and leveraging finger orientation for interaction with direct-touch surfaces," Proc. UIST 2009, pp.23-32, 2009.

[19] D. Schmidt, F. Block, and H. Gellersen, "A comparison of direct and indirect multi-touch input for large surfaces," Proc. ISFT 2009, vol.5726, pp.583-594, 2009.

[20] K. Pfeuffer, J. Alexander, M. Chong, Y. Zhang, and H. Gellersen, "Gaze-Shifting: direct-indirect input with pen and touch modulated by gaze," Proc. UIST 2015, pp.373-383, 2015.

[21] S. Voelker, A. Matviienko, J. Schöning, and J. Borchers, "Combining direct and indirect touch input for interactive workspaces using gaze input," Proc. SUI 2015, pp.79-88, 2015.

[22] H. Palleis and H. Hussmann, "Indirect 2D touch panning: how does it affect spatial memory and navigation performance?," Proc. CHI 2016, pp.1947-1951, 2016.

[23] K. Pfeuffer and H. Gellersen, "Gaze and touch interaction on tablets," Proc. UIST 2016, pp.301-311, 2016.

[24] H. Palleis, J. Wagner, and H. Hussmann, "Novel indirect touch input techniques applied to finger-forming 3D models," Proc. AVI 2016, pp.228-235, 2016.

[25] H. Palleis and V. Niedermeier, "Exploring two-handed indirect multi-touch input for a docking task," Proc. AVI 2016, pp.36-39, 2016.

[26] L. Findlater, K. Moffatt, J. Froehlich, M. Malu, and J. Zhang, "Comparing touchscreen and mouse input performance by people with and without upper body motor impairments," Proc. CHI 2017, pp.6056-6061, 2017.

[27] P.M. Fitts, "The information capacity of the human motor system in controlling the amplitude of movement," Experimental Psychology, vol.47, no.6, pp.381-391, 1954.

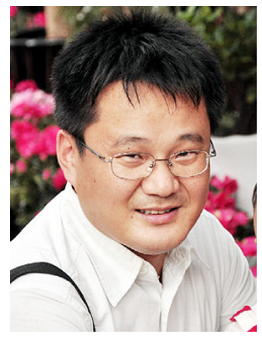

Yizhong Xin received B.S. and M.S. degrees in Computer Science from Shenyang University of Technology, China in 1997 and 2004, respectively and received Ph.D degree in Computer Science from Kochi University of Technology, Japan in 2010. In 2005-2006, he stayed at Aalen University, Germany to do research on Information Security. He is now a professor and doctoral advisor in the School of Information Science and Engineering at Shenyang University of Technology, China. 


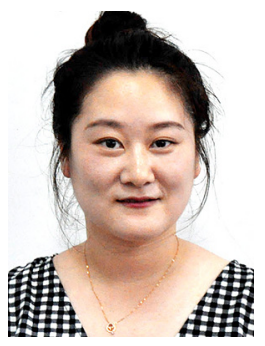

Ruonan Liu received B.S. and M.S. degrees in Computer Science from Shenyang University of Technology, China in 2015 and 2018, respectively. She is now a Doctoral candidate of Shenyang University of Technology, China.

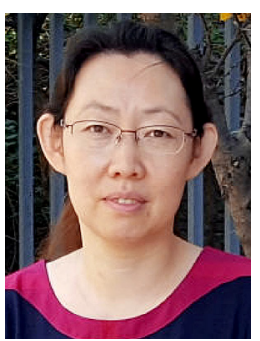

Yan Li received B.S. and M.S. degrees in Computer Science from Shenyang University of Technology, China in 1998 and 2007, respectively. She is now a Lecturer in the School of Management and News Dissemination at Shenyang Sport University, China. 